\title{
Rare Three-Dimensional Uranyl-Biphenyl-3,3' '-disulfonyl-4,4'-dicarboxylate Frameworks: Crystal Structures, Proton Conductivity, and Luminescence
}

\author{
Dan-Dan Liu, ${ }^{\dagger}$ Yu-Ling Wang, ${ }^{*},{ }^{\dagger}$ Feng Luo, ${ }^{\ddagger}$ and Qing-Yan Liu $*, \dagger$ \\ ${ }^{\dagger}$ College of Chemistry and Chemical Engineering, Jiangxi Normal University, Nanchang, Jiangxi 330022, P. R. \\ China.
}

${ }^{*}$ College of Biology, Chemistry and Material Science, East China Institute of Technology, Nanchang, Jiangxi 34400, P. R. China.

\section{Supporting Information}

Physical Measurements. IR (KBr pellets) spectra were recorded in the $400-4000 \mathrm{~cm}^{-1}$ range using a Perkin-Elmer Spectrum One FT-IR spectrometer. Elemental analyses were carried out on Elementar Perkin-Elmer 2400CHN microanalyzer. Thermogravimetric analyses were carried out on a PE Diamond TG/DTA unit at a heating rate of $10{ }^{\circ} \mathrm{C} / \mathrm{min}$ under a nitrogen atmosphere. Powder X-ray diffraction patterns were performed on a Rigaku Miniflex 600 powder diffractometer using $\mathrm{Cu}-\mathrm{K} \alpha$ radiation $(\lambda=1.5418 \AA)$. The proton conductivity data was interrogated by the conventional quasi-four-probe method using gold paste and gold wires $(50 \mu \mathrm{m}$ diameter $)$ with an Impedance/Gain-Phase Analyzer (Solartron SI 1260) under different environmental conditions. The Electrochemical Impedance Spectroscopy (EIS) was taken by applying $100 \mathrm{mV}$ AC amplitude voltage over the frequency from 1 to $1 \times 10^{6} \mathrm{~Hz}$ in 10 steps on a logarithmic scale. The impedance results were analyzed in ZView2 (Scribner Associates), which was used to generate Nyquist plots 
with the real parts $\left(Z^{\prime}\right)$ as the abscissa and the imaginary part $\left(Z^{\prime \prime}\right)$ as the ordinate. The pellets were exposed to controlled humidity and temperature environments which was performed using an XK-CTS80Z incubator (Shenzhen selenium control testing equipment corp.). Fluorescent spectra were measured at room temperature with a single-grating Edinburgh EI980 fluorescence spectrometer.
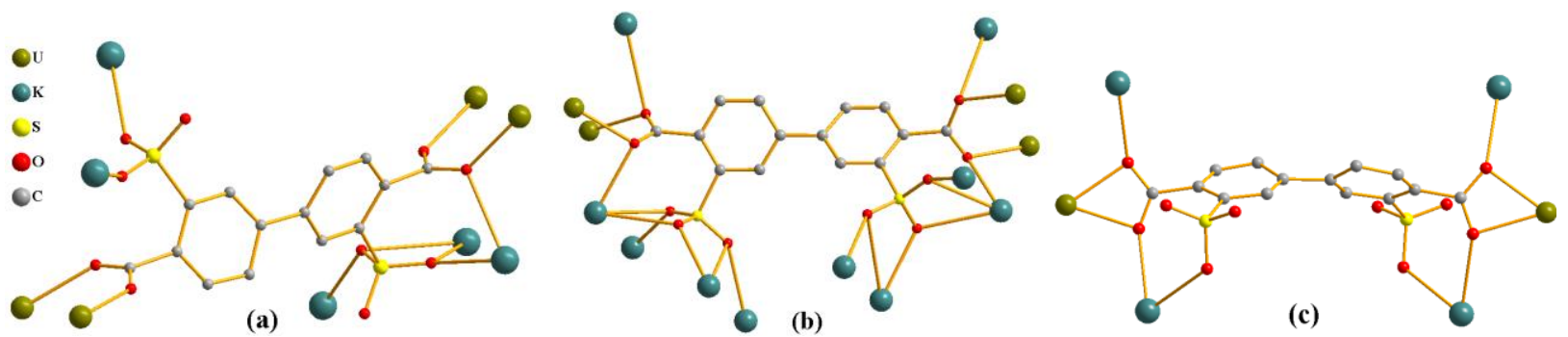

Scheme S1 Coordination modes of the BPDSDC ${ }^{4-}$ ligand in 1-3.

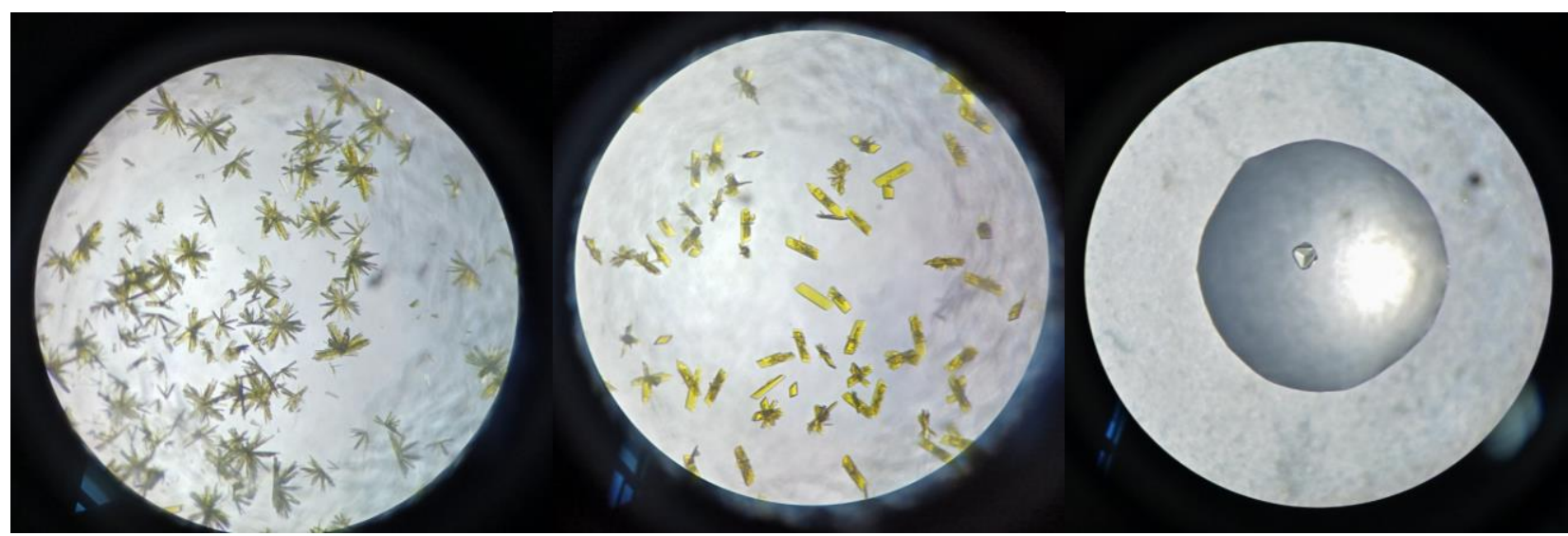

Figure S1. The photographs of crystals for $\mathbf{1}$ (left), 2 (middle) and $\mathbf{3}$ (right).

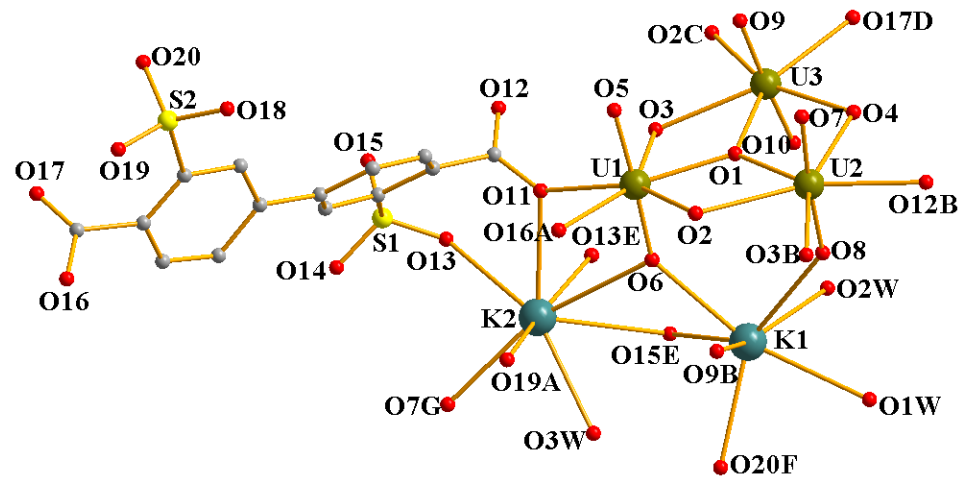

Figure S2. The coordination environments of $\mathrm{UO}^{2+}$ and $\mathrm{K}^{+}$ions in $\mathbf{1}$. Symmetry codes: $\mathrm{A}-x+1,-y$ $+1,-z+1 ; \mathrm{B}-x+3 / 2, y+1 / 2,-z+1 / 2 ; \mathrm{C}-x+3 / 2, y-1 / 2,-z+1 / 2 ; \mathrm{D} x+1 / 2,-y+1 / 2, z-1 / 2$; $\mathrm{E}-x+3 / 2,-y+1 / 2,-z+1 ; \mathrm{F} x+1 / 2, y+1 / 2, z ; \mathrm{G} x,-y+1, z+1 / 2$. 


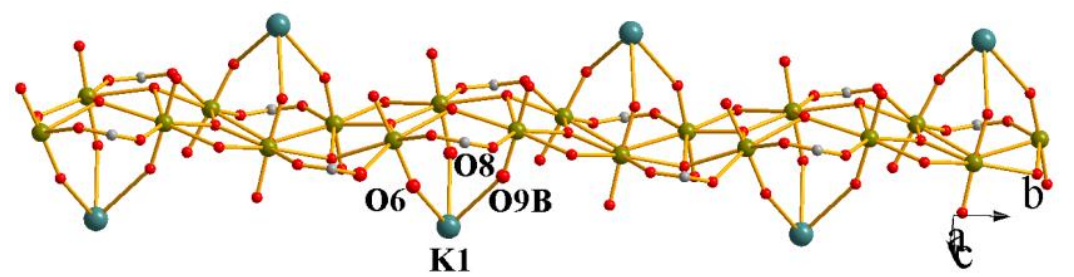

Figure S3. The $1 \mathrm{D}$ ribbon is capped by the $\mathrm{K} 1$ ion through the $\mathrm{K}-\mathrm{O}$ bonds with the $\mathrm{O}$ atoms of $\mathrm{UO}^{2+}$ units in $\mathbf{1 .}$

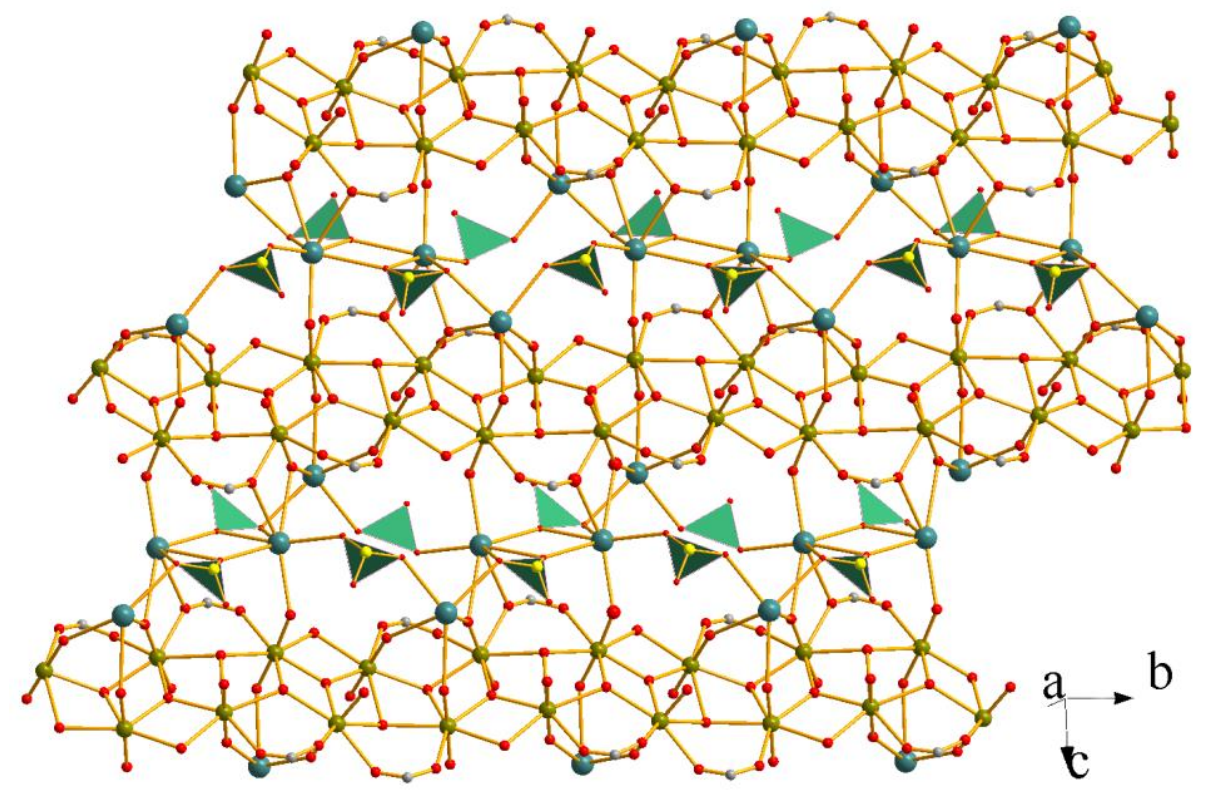

Figure S4. The 2D layer in 1 (The sulfonate groups represent as polyhedrons).

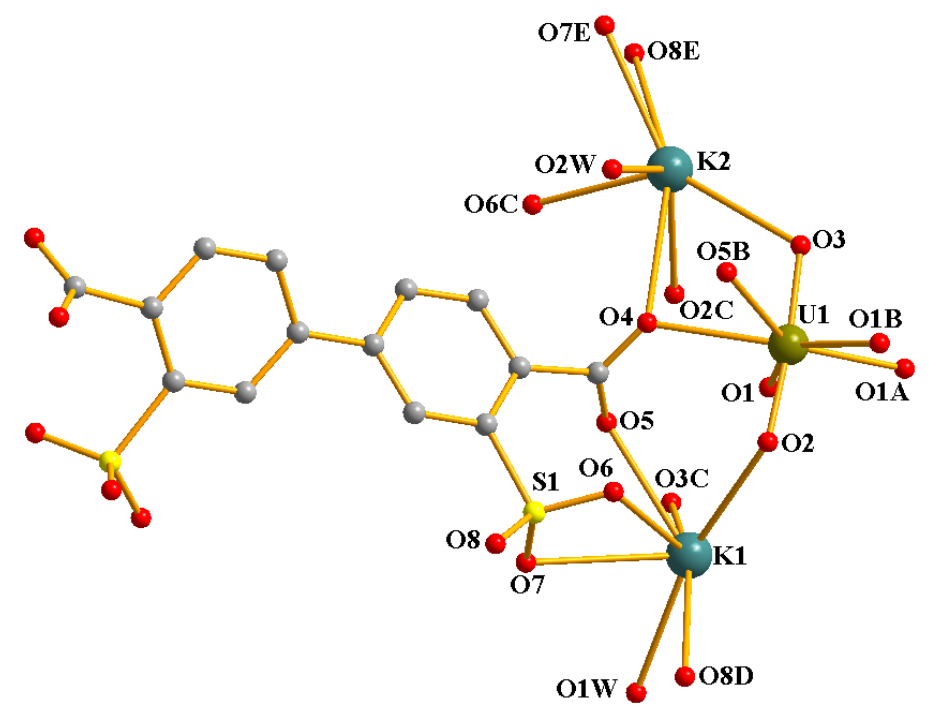

Figure S5. The coordination environments of $\mathrm{UO}^{2+}$ and $\mathrm{K}^{+}$ions in 2 . Symmetry codes: $\mathrm{A}-x+1,-y$ $+1,-z+1 ; \mathrm{B} x,-y+1, z+1 / 2 ; \mathrm{C} x,-y+1, z-1 / 2 ; \mathrm{D}-x+1 / 2,-y+1 / 2,-z+1 ; \mathrm{E}-x+1 / 2, y+$ $1 / 2,-z+1 / 2 ; \mathrm{F}-x+1, y,-z+3 / 2$. 


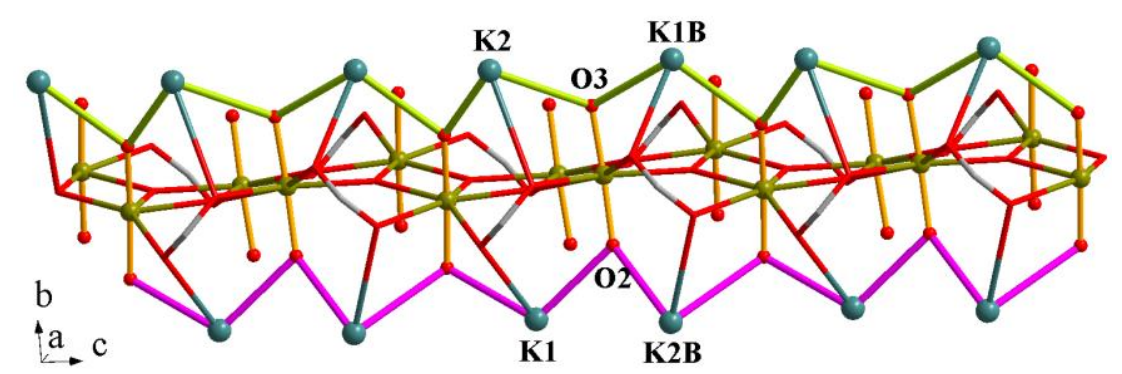

Figure S6. The oxygen atoms $(\mathrm{O} 2$ and $\mathrm{O} 3)$ of the $\mathrm{UO}_{2}{ }^{2+}$ units link the $\mathrm{K} 1$ and $\mathrm{K} 2$ atoms to give the infinite chains in $\mathbf{2}$ (here only showing two 1D chains).

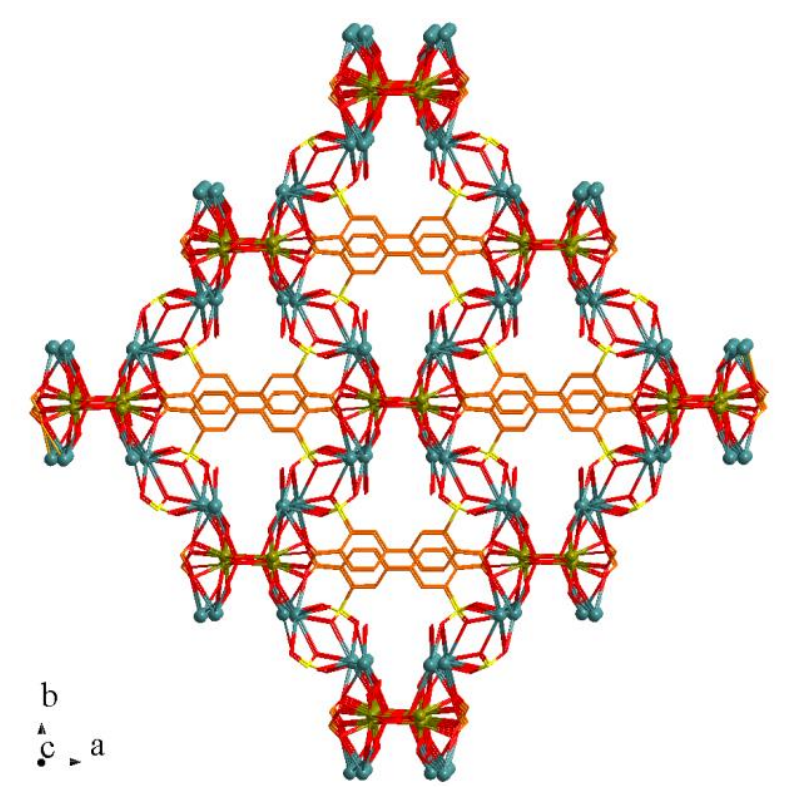

Figure S7. 3D structure of $\mathbf{2}$ shows the coordinated water molecules point to the pores.

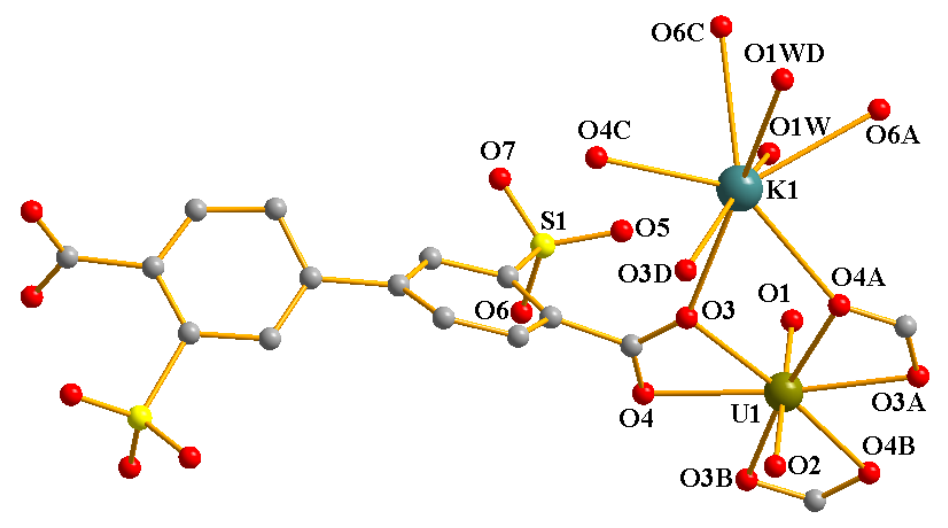

Figure S8. The coordination environments of $\mathrm{UO}^{2+}$ and $\mathrm{K}^{+}$ions in 3. Symmetry codes: A $z, x, y$; $\mathrm{B}$, $z, x ; \mathrm{C} y-1 / 4,-x+3 / 4, z+1 / 4 ; \mathrm{D} z-1 / 4,-y+3 / 4, x+1 / 4$. 


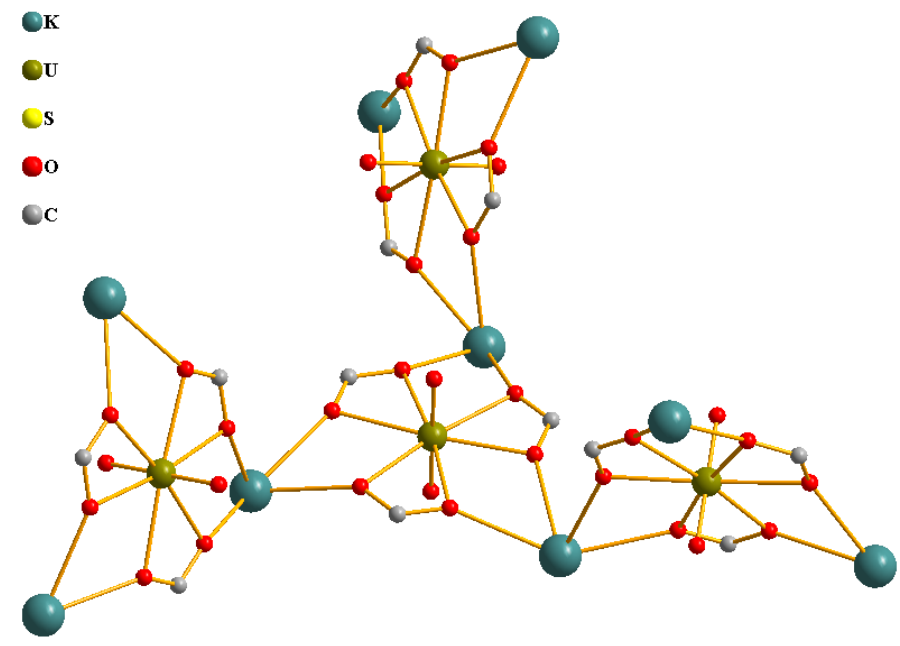

Figure S9. Each $\left[\left(\mathrm{UO}_{2}\right)(\mathrm{COO})_{3}\right]$ unit is linked to three $\left[\left(\mathrm{UO}_{2}\right)(\mathrm{COO})_{3}\right]$ units through $\mathrm{K}^{+}$ions in 3 .

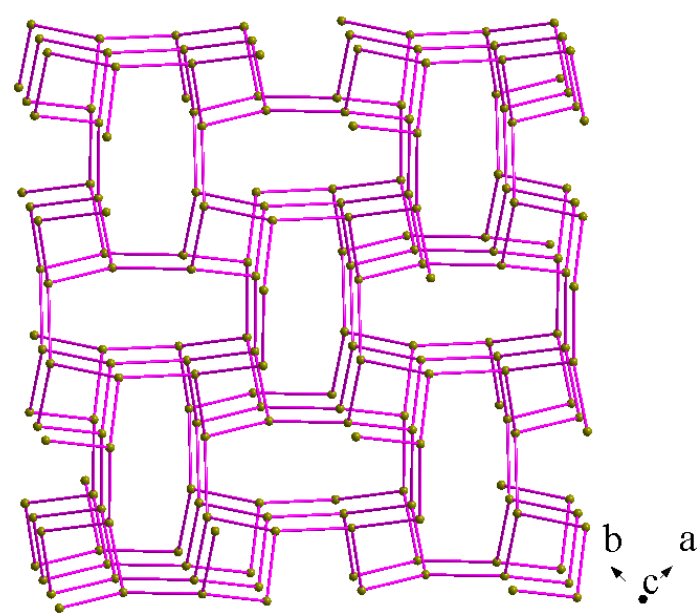

Figure S10. The srs topological net of 3D $\left[\mathrm{K}_{1.5}\left(\mathrm{UO}_{2}\right)(\mathrm{COO})_{3}\right]_{\mathrm{n}}$ substructure in 3 (The mononuclear $\left[\left(\mathrm{UO}_{2}\right)(\mathrm{COO})_{3}\right]$ unit is defined as a node). 


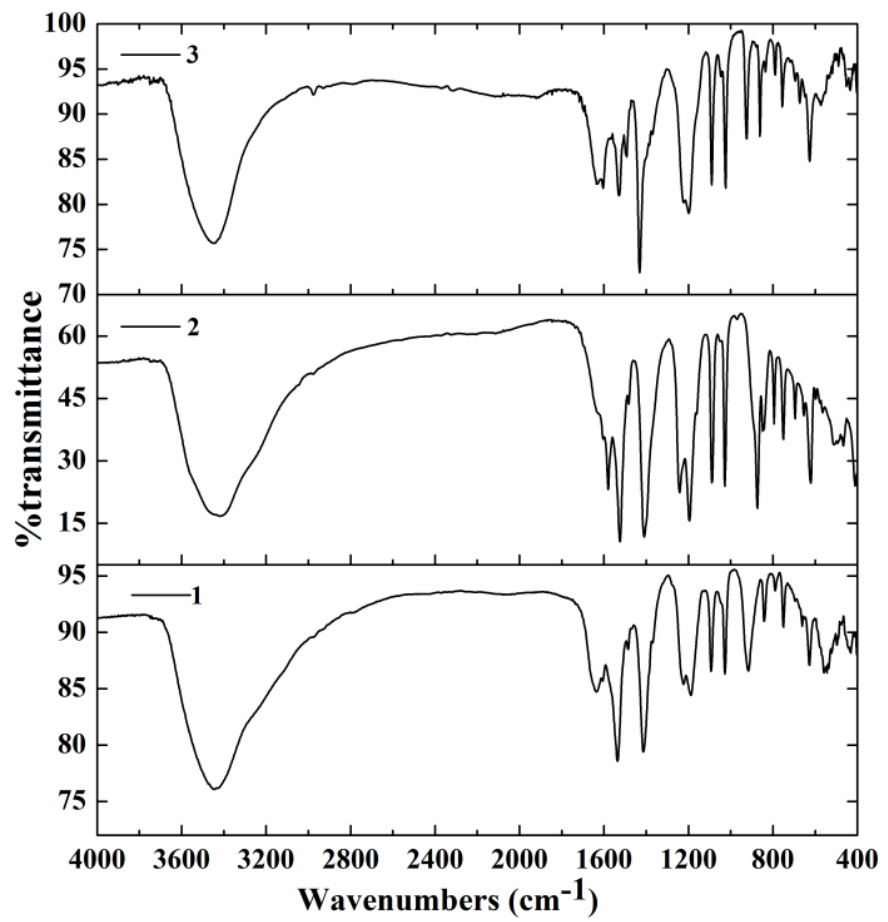

Figure S11. IR spectra of 1-3.
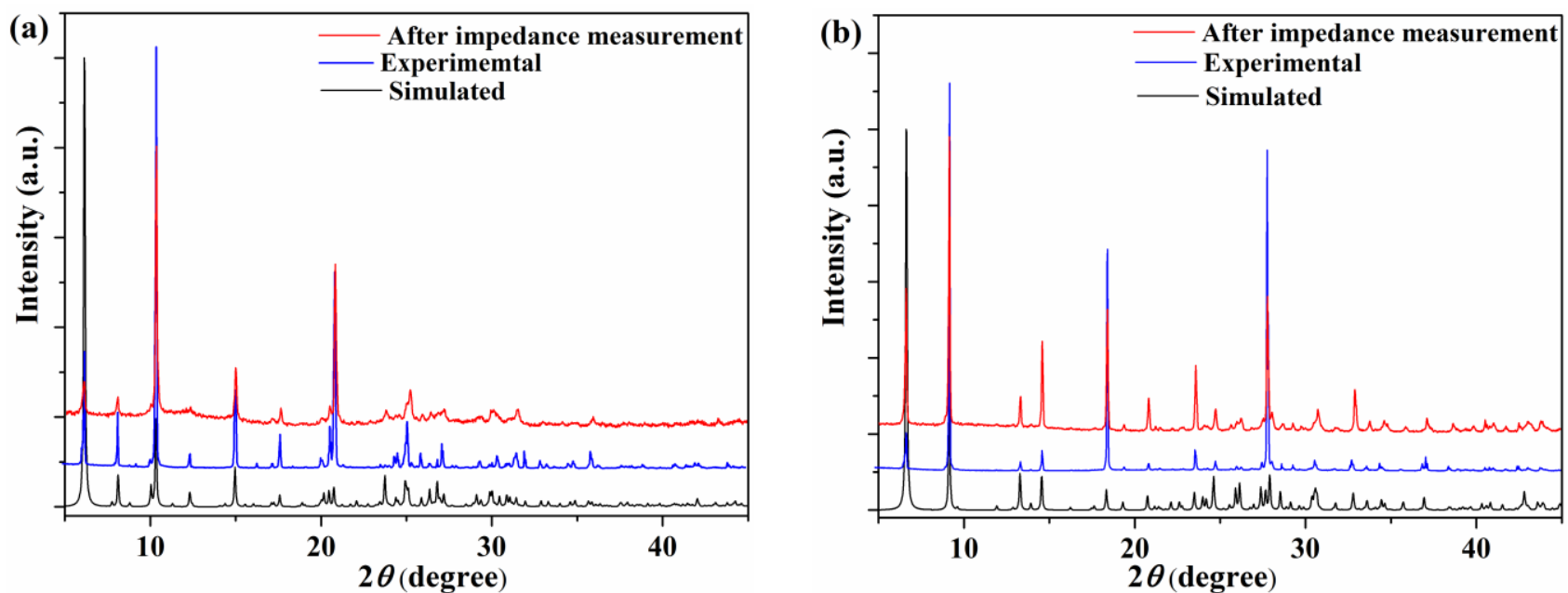

Figure S12. PXRD patterns for 1 (a) and 2 (b).

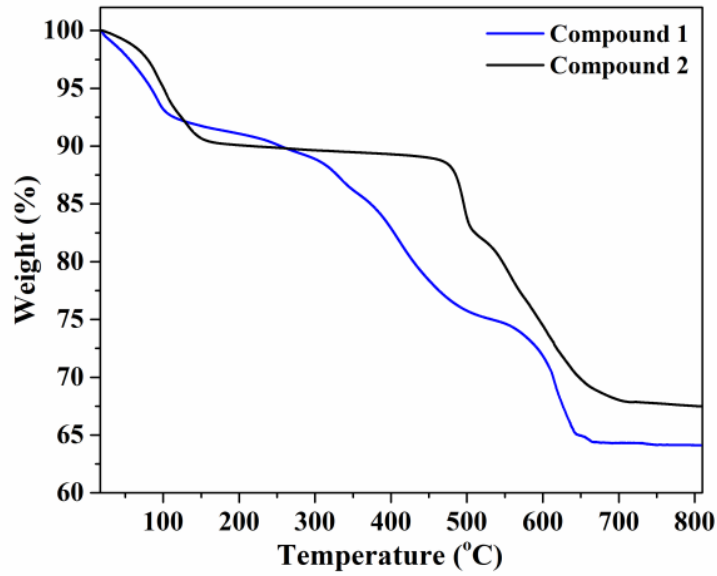

Figure S13. TGA curves for $\mathbf{1}$ and 2. 


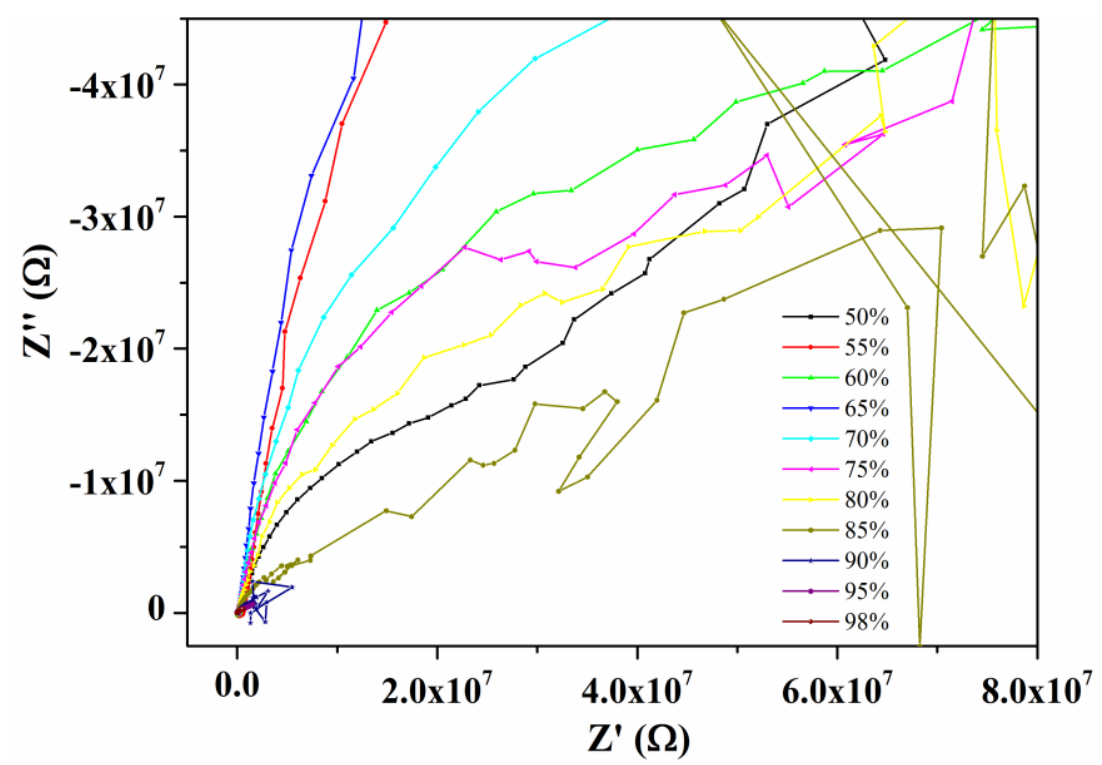

Figure S14. Impedance plots for 1 at $85^{\circ} \mathrm{C}$ and various humidities.

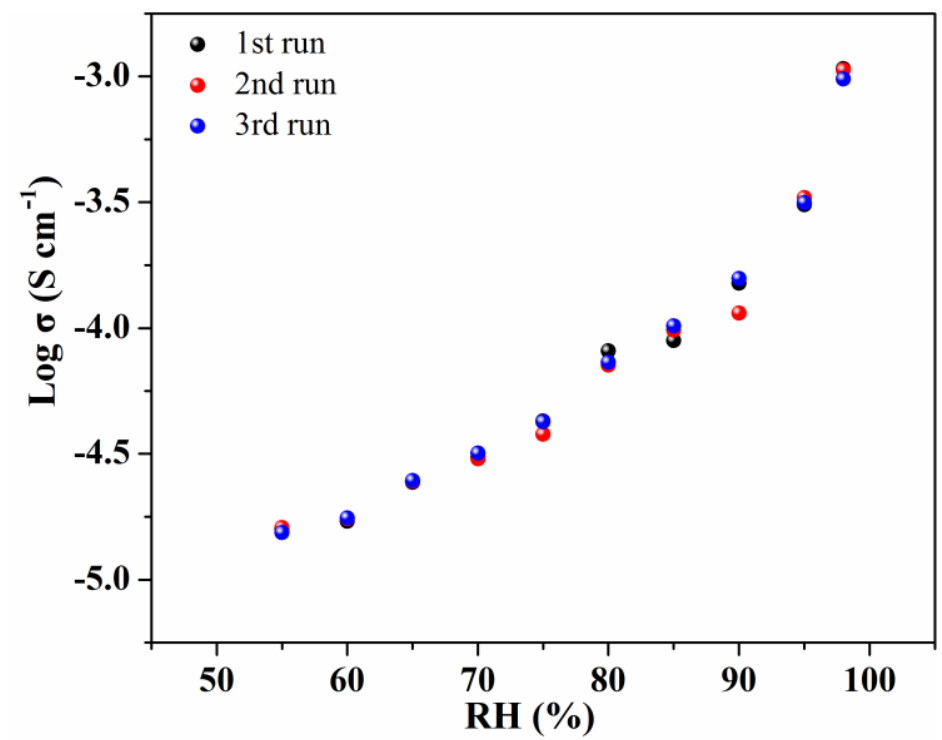

Figure S15. Plots of $\log (\sigma) v s \mathrm{RH}$ of 2 at $85^{\circ} \mathrm{C}$ with three cycles. 


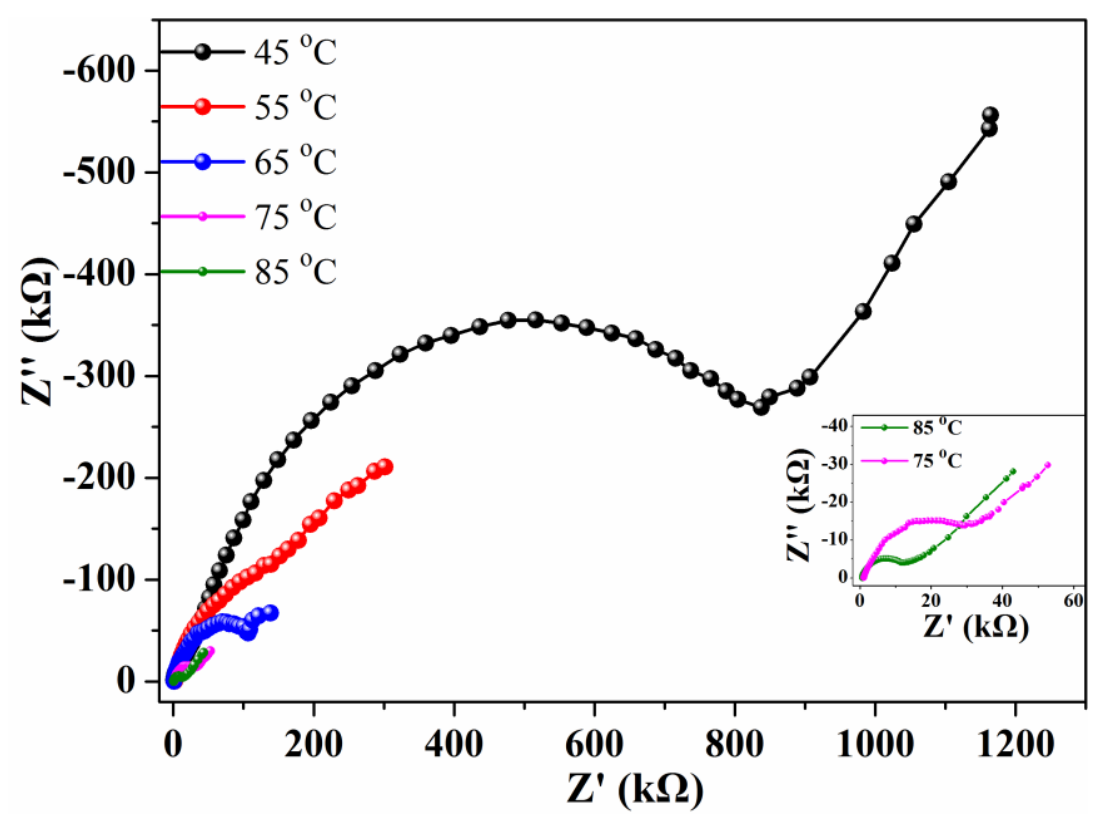

Figure S16. Impedance plots for 2 at $98 \% \mathrm{RH}$ and various temperatures (Inset: enlarged curves at 85 and $75^{\circ} \mathrm{C}$ ).

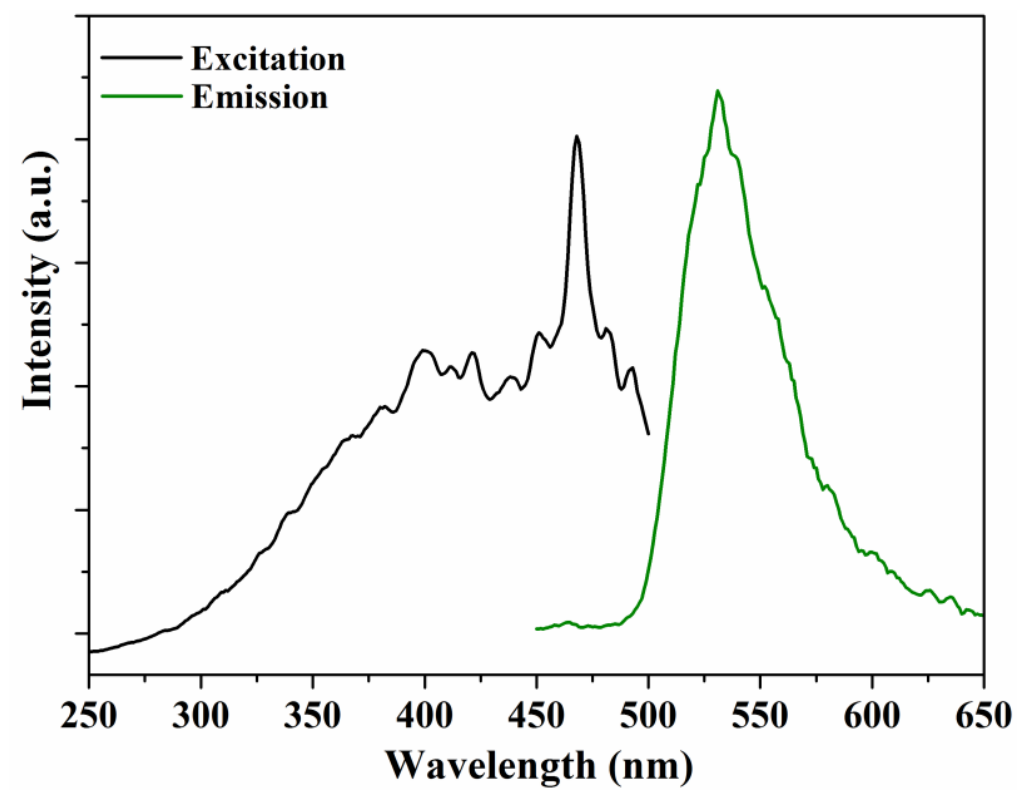

Figure S17. Solid-state excitation and emission spectra $\left(\lambda_{\mathrm{ex}}=420 \mathrm{~nm}\right)$ of 1 at room temperature. 


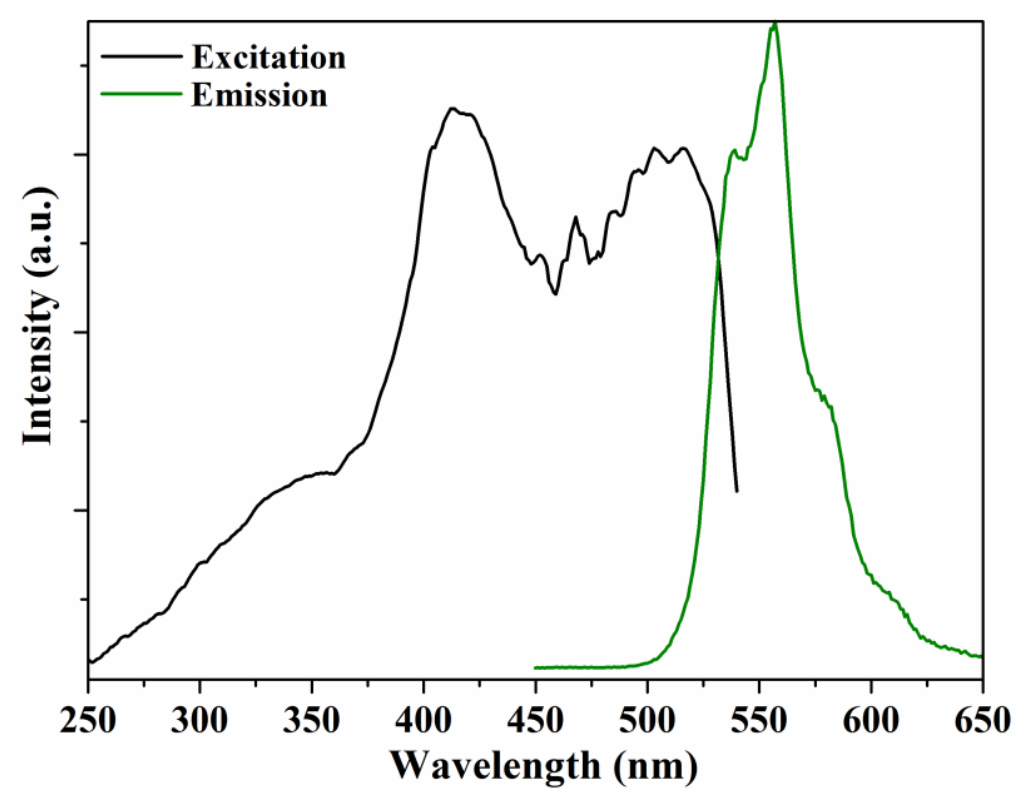

Figure S18. Solid-state excitation and emission spectra $\left(\lambda_{\mathrm{ex}}=420 \mathrm{~nm}\right)$ of 2 at room temperature.

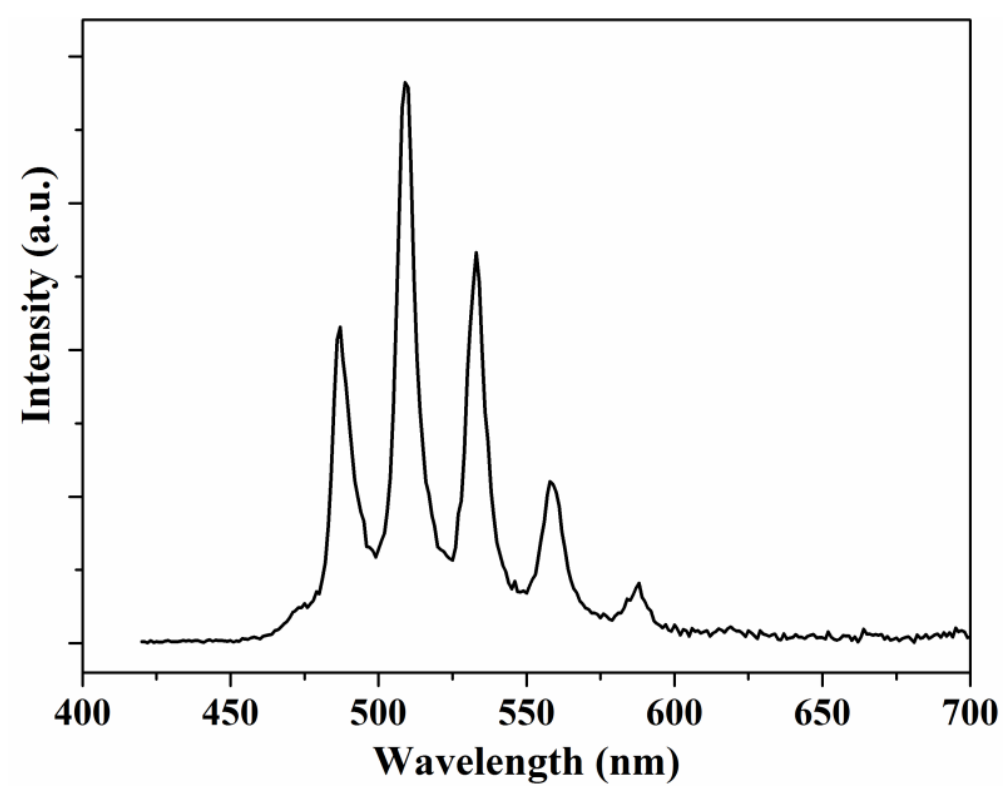

Figure S19. Solid-state emission spectrum $\left(\lambda_{\mathrm{ex}}=420 \mathrm{~nm}\right)$ of $\mathrm{UO}_{2}\left(\mathrm{NO}_{3}\right)_{2} \cdot 6 \mathrm{H}_{2} \mathrm{O}$ at room temperature.

Table S1. Selected Bond Lengths $(\AA)$ of $\mathbf{1}-\mathbf{3}^{a}$

\begin{tabular}{llllll}
\hline $\mathbf{1}$ & \multicolumn{3}{l}{$\mathbf{3}$} & \\
\hline $\mathrm{U} 1-\mathrm{O} 5$ & $1.782(11)$ & $\mathrm{U} 1-\mathrm{O} 3$ & $1.816(5)$ & $\mathrm{U} 1-\mathrm{O} 1$ & $1.69(2)$ \\
$\mathrm{U} 1-\mathrm{O} 6$ & $1.785(11)$ & $\mathrm{U} 1-\mathrm{O} 2$ & $1.811(5)$ & $\mathrm{U} 1-\mathrm{O} 2$ & $1.78(2)$ \\
$\mathrm{U} 1-\mathrm{O} 1$ & $2.216(11)$ & $\mathrm{U} 1-\mathrm{O} 1$ & $2.253(5)$ & $\mathrm{U} 1-\mathrm{O} 3$ & $2.439(9)$ \\
$\mathrm{U} 1-\mathrm{O} 11$ & $2.370(11)$ & $\mathrm{U} 1-\mathrm{O} 1 \mathrm{~A}$ & $2.264(5)$ & $\mathrm{U} 1-\mathrm{O} 3 \mathrm{~A}$ & $2.439(9)$ \\
$\mathrm{U} 1-\mathrm{O} 16 \mathrm{~A}$ & $2.391(11)$ & $\mathrm{U} 1-\mathrm{O} 1 \mathrm{~B}$ & $2.269(5)$ & $\mathrm{U} 1-\mathrm{O} 3 \mathrm{~B}$ & $2.439(9)$
\end{tabular}




\begin{tabular}{|c|c|c|c|c|c|}
\hline $\mathrm{U} 1-\mathrm{O} 2$ & $2.422(10)$ & $\mathrm{U} 1-\mathrm{O} 4$ & $2.505(5)$ & $\mathrm{U} 1-\mathrm{O} 4$ & $2.479(9)$ \\
\hline $\mathrm{U} 1-\mathrm{O} 3$ & $2.495(9)$ & $\mathrm{U} 1-\mathrm{O} 5 \mathrm{~B}$ & $2.555(5)$ & $\mathrm{U} 1-\mathrm{O} 4 \mathrm{~A}$ & $2.479(9)$ \\
\hline $\mathrm{U} 2-\mathrm{O} 8$ & $1.802(11)$ & $\mathrm{K} 1-\mathrm{O} 5$ & $2.766(6)$ & $\mathrm{U} 1-\mathrm{O} 4 \mathrm{~B}$ & $2.479(9)$ \\
\hline $\mathrm{U} 2-\mathrm{O} 7$ & $1.794(11)$ & $\mathrm{K} 1-\mathrm{O} 8 \mathrm{D}$ & $2.811(7)$ & $\mathrm{K} 1-\mathrm{O} 3$ & $2.753(11)$ \\
\hline $\mathrm{U} 2-\mathrm{O} 1$ & $2.204(10)$ & $\mathrm{K} 1-\mathrm{O} 2$ & $2.828(6)$ & $\mathrm{K} 1-\mathrm{O} 3 \mathrm{D}$ & $2.753(11)$ \\
\hline $\mathrm{U} 2-\mathrm{O} 4$ & $2.372(10)$ & $\mathrm{K} 1-\mathrm{O} 1 \mathrm{~W}$ & $2.819(14)$ & $\mathrm{K} 1-\mathrm{O} 4 \mathrm{~A}$ & $2.781(10)$ \\
\hline $\mathrm{U} 2-\mathrm{O} 3 \mathrm{~B}$ & $2.427(9)$ & $\mathrm{K} 1-\mathrm{O} 3 \mathrm{C}$ & $2.896(6)$ & $\mathrm{K} 1-\mathrm{O} 4 \mathrm{C}$ & $2.781(10)$ \\
\hline $\mathrm{U} 2-\mathrm{O} 12 \mathrm{~B}$ & $2.480(11)$ & $\mathrm{K} 1-\mathrm{O} 6$ & $2.976(7)$ & $\mathrm{K} 1-\mathrm{O} 6 \mathrm{C}$ & $2.876(19)$ \\
\hline $\mathrm{U} 2-\mathrm{O} 2$ & $2.573(10)$ & $\mathrm{K} 1-\mathrm{O} 7$ & $3.011(8)$ & $\mathrm{K} 1-\mathrm{O} 6 \mathrm{~A}$ & $2.876(19)$ \\
\hline $\mathrm{U} 3-\mathrm{O} 10$ & $1.770(11)$ & $\mathrm{K} 2-\mathrm{O} 2 \mathrm{~W}$ & $2.703(9)$ & $\mathrm{K} 1-\mathrm{O} 1 \mathrm{~W}$ & $3.39(4)$ \\
\hline U3-O9 & $1.793(11)$ & $\mathrm{K} 2-\mathrm{O} 6 \mathrm{C}$ & $2.716(6)$ & $\mathrm{K} 1-\mathrm{O} 1 \mathrm{WD}$ & $3.39(4)$ \\
\hline U3-O1 & $2.232(10)$ & $\mathrm{K} 2-\mathrm{O} 2 \mathrm{C}$ & $2.759(6)$ & & \\
\hline U3-O4 & $2.394(10)$ & $\mathrm{K} 2-\mathrm{O} 7 \mathrm{E}$ & $2.774(7)$ & & \\
\hline $\mathrm{U} 3-\mathrm{O} 2 \mathrm{C}$ & $2.425(9)$ & $\mathrm{K} 2-\mathrm{O} 3$ & $3.006(5)$ & & \\
\hline U3-O17D & $2.416(11)$ & $\mathrm{K} 2-\mathrm{O} 4$ & $3.184(6)$ & & \\
\hline $\mathrm{U} 3-\mathrm{O} 3$ & $2.551(10)$ & $\mathrm{K} 2-\mathrm{O} 8 \mathrm{E}$ & $3.382(9)$ & & \\
\hline $\mathrm{K} 1-\mathrm{O} 20 \mathrm{~F}$ & $2.715(18)$ & & & & \\
\hline $\mathrm{K} 1-\mathrm{O} 6$ & $2.732(12)$ & & & & \\
\hline $\mathrm{K} 1-\mathrm{O} 15 \mathrm{E}$ & $2.840(13)$ & & & & \\
\hline $\mathrm{K} 1-\mathrm{O} 8$ & $2.882(13)$ & & & & \\
\hline $\mathrm{K} 1-\mathrm{O} 1 \mathrm{~W}$ & $2.84(3)$ & & & & \\
\hline $\mathrm{K} 1-\mathrm{O} 9 \mathrm{~B}$ & $2.876(12)$ & & & & \\
\hline $\mathrm{K} 1-\mathrm{O} 2 \mathrm{~W}$ & $3.38(4)$ & & & & \\
\hline $\mathrm{K} 2-\mathrm{O} 19 \mathrm{~A}$ & $2.634(16)$ & & & & \\
\hline $\mathrm{K} 2-\mathrm{O} 13 \mathrm{E}$ & $2.711(15)$ & & & & \\
\hline $\mathrm{K} 2-\mathrm{O} 7 \mathrm{G}$ & $2.760(13)$ & & & & \\
\hline $\mathrm{K} 2-\mathrm{O} 3 \mathrm{~W}$ & $2.796(18)$ & & & & \\
\hline $\mathrm{K} 2-\mathrm{O} 11$ & $2.803(11)$ & & & & \\
\hline $\mathrm{K} 2-\mathrm{O} 13$ & $2.818(14)$ & & & & \\
\hline $\mathrm{K} 2-\mathrm{O} 6$ & $3.034(12)$ & & & & \\
\hline $\mathrm{K} 2-\mathrm{O} 15 \mathrm{E}$ & $3.172(16)$ & & & & \\
\hline
\end{tabular}


${ }^{a}$ symmetry Codes, For 1 : $\mathrm{A}-x+1,-y+1,-z+1$; $\mathrm{B}-x+3 / 2, y+1 / 2,-z+1 / 2 ; \mathrm{C}-x+$ $3 / 2, y-1 / 2,-z+1 / 2 ; \mathrm{D} x+1 / 2,-y+1 / 2, z-1 / 2 ; \mathrm{E}-x+3 / 2,-y+1 / 2,-z+1 ; \mathrm{F} x+1 / 2, y+$ $1 / 2, z ; \mathrm{G} x,-y+1, z+1 / 2$. For $2: \mathrm{A}-x+1,-y+1,-z+1 ; \mathrm{B} x,-y+1, z+1 / 2 ; \mathrm{C} x,-y+$ $1, z-1 / 2 ; \mathrm{D}-x+1 / 2,-y+1 / 2,-z+1 ; \mathrm{E}-x+1 / 2, y+1 / 2,-z+1 / 2$. For 3: A $z, x, y ; \mathrm{B} y$, $z, x ; \mathrm{C} y-1 / 4,-x+3 / 4, z+1 / 4 ; \mathrm{D} z-1 / 4,-y+3 / 4, x+1 / 4$. 